\title{
Sequenciamento de Ações Pedagógicas baseadas na Taxonomia de Bloom usando Planejamento Automatizado apoiado por Algoritmo Genético
}

\author{
Title: Sequencing of Pedagogical Actions based on Bloom's Taxonomy using Automated \\ Planning supported by Genetic Algorithm
}

\author{
Newarney Torrezão da Costa \\ Instituto Federal de Educação, Ciência e \\ Tecnologia Goiano (IF Goiano) \\ Iporá - GO-Brasil \\ newarney.costa@ifgoiano.edu.br
}

\author{
Márcia Aparecida Fernandes \\ Faculdade de Computação \\ Universidade Federal de Uberlândia (UFU) \\ Uberlândia - MG-Brasil \\ marcia@ufu.br
}

\begin{abstract}
Resumo
Este trabalho investigou o planejamento em Inteligência Artificial para o sequenciamento de ações pedagógicas, de acordo com o perfil do estudante. As ações representaram o processo cognitivo dado pela Taxonomia de Bloom (TB) e o perfil do estudante foi determinado conforme o Revised Approaches to Studying Inventory (RASI). A fim de medir a adequação de uma sequência ao perfil RASI do estudante, foi necessário mapear estas duas teorias, sendo este mapeamento uma das contribuições deste estudo. Assim, o sequenciamento de ações foi formulado como um problema de otimização e implementado através de Algoritmo Genético. A proposição da função a ser otimizada para este problema é também uma contribuição, desde que estabelecer critérios para avaliar aspectos pedagógicos tem sido um desafio para Informática na Educação. Experimentos realizados contaram com 41 participantes que responderam ao questionário RASI e, após terem recebido e analisado as respectivas sequências de ações determinadas pelo planejador proposto neste trabalho, também responderam a um questionário de satisfação em relação à sequência. Os resultados obtidos se mostraram promissores, demonstrando a viabilidade da pesquisa.

Palavras-Chave: Planejamento em Inteligência artificial; Taxonomia dos objetivos educacionais; RASI; Algoritmo genético.
\end{abstract}

\begin{abstract}
This work investigated the Artificial Intelligence planning for pedagogical actions sequencing, according to the student's profile. The actions represented the cognitive process given by Bloom's Taxonomy (BT) and the student profile was modeled by the Revised Approaches to Studying Inventory (RASI). To measure the suitability of a sequence to the student's profile, it was necessary to map these two theories, this mapping being one of the contributions of this study. Thus, the sequencing of actions was formulated as an optimization problem and developed through Genetic Algorithm. The proposition of the function to be optimized for this problem is also a contribution, since establishing criteria to evaluate pedagogical aspects has been a challenge for Informatics in Education. Experiments carried out had 41 participants who answered the RASI inventory and, after receiving and analyzing the sequences of actions generated by the planner proposed in this work, they also answered a satisfaction questionnaire about the sequence. The results obtained can be considered promising, demonstrating the feasibility of the research.
\end{abstract}

Keywords: Artificial Intelligence Planning; Taxonomy of educational goals; RASI; Genetic algorithm.

Cite as: Costa, N. T., \& Fernandes, M. A. (2021). Sequencing of Pedagogical Actions based on Bloom's Taxonomy using Automated Planning supported by Genetic Algorithm (Sequenciamento de Ações Pedagógicas baseadas na Taxonomia de Bloom usando Planejamento Automatizado apoiado por Algoritmo Genético). Brazilian Journal of Computers in Education (Revista Brasileira de Informática na Educação - RBIE), 29, 485-501. DOI: 10.5753/RBIE.2021.29.0.485 


\section{Introdução}

Em informática na educação, é comum observar a utilização de técnicas computacionais que promovem intervenções pedagógicas relacionadas ao ensino, à aprendizagem, ou ambos. Nesse sentido, o sequenciamento pedagógico pode ser utilizado com o objetivo de auxiliar no processo de aprendizagem (Silva, Direne, Marczal, Borille, \& Guimarães, 2018), (Júnior, Araújo, \& Dorça, 2020). Tais intervenções podem contar com técnicas de Inteligência Artificial (IA). Dentre essas, o Planejamento Automatizado (PA) se destaca, pois determina ações que permitam o cumprimento eficaz de um objetivo, segundo critérios estabelecidos (Costa, Pereira Júnior, \& Fernandes, 2019)

Como relatado em (Costa, Pereira Junior, Araújo, \& Fernandes, 2019), a partir do ano de 2016 observa-se a evolução de trabalhos que utilizam técnicas de PA com o propósito supracitado. Entretanto, nota-se que a Planning Domain Definition Language (PDDL), linguagem comumente utilizada para o PA, pode não ser uma opção viável no contexto de sequenciamento automático de ações pedagógicas, pois à medida que o modelo do estudante é refinado e são utilizados mais parâmetros para auxiliar no planejamento, tornam-se mais limitadas as heurísticas que possibilitam a busca no espaço de soluções (de Lima, Feijó, \& Furtado, 2019).

O uso de algoritmos baseados em computação bioinspirada como apoio ao PA para realização de sequenciamento de ações pedagógicas, parte do princípio de que esse tipo de problema pode ser interpretado como um problema de otimização (Hssina, 2019). Nesse sentido, o Algoritmo Genético é uma das técnicas que pode apresentar vantagens em relação ao uso da PDDL, visto que essa é complexa e aquele apresenta maior flexibilidade para lidar com uma maior variedade de heurísticas em diversos cenários, como apresentado em (Brie \& Morignot, 2005).

A modelagem das ações pedagógicas é outro fator decisivo no processo de sequenciamento. Nesse sentido, a definição de ações que representem o processo cognitivo pode prover melhoria maneira como ocorre a aprendizagem, uma vez que o foco desse sequenciamento passa a ser perfil do estudante (Costa, Pereira Junior, Araújo, \& Fernandes, 2019). A Taxonomia de Bloom revisada, neste trabalho referenciada apenas como Taxonomia de Bloom (TB), é uma alternativa, pois torna viável a modelagem de tais ações a partir do domínio cognitivo do estudante (Krathwohl, 2002).

A observação de características cognitivas do estudante é fundamental para o sucesso no processo de personalização do sequenciamento pedagógico. Diversas alternativas podem ser utilizadas com tal finalidade, por exemplo em (Garrido, Morales, \& Serina, 2016), as preferências modeladas por meio de estilos de aprendizagem são utilizadas. Os metadados de Ambientes Virtuais de Aprendizagem (AVAs) também podem ser utilizados com esse propósito, como observado em (Pireva \& Kefalas, 2018). Já em (Limongelli \& Sciarrone, 2014), o estado cognitivo do estudante, representado por meio da Taxonomia de Bloom, é utilizado. Outras características podem ser utilizadas individualmente ou associadas, de modo a compor um conjunto de atributos do estudante. Uma alternativa explorada para recomendações pedagógicas pode ser observada em (Costa, Pereira Júnior, \& Fernandes, Recomendação de Ações Pedagógicas Utilizando Planejamento Automático e Taxonomia Digital de Bloom, 2019), onde o Approaches and Study Skills Inventory for Students (ASSIST) é utilizado para compor o perfil do estudante no processo de recomendação de ações pedagógicas. Nesse trabalho, tal abordagem se mostra promissora, pois apresenta uma perspectiva relacionada à Taxonomia de Bloom.

Assim, neste trabalho é proposto o sequenciamento de ações pedagógicas estruturadas de acordo com a Taxonomia de Bloom. Essa abordagem permite modelar as ações sob a ótica do domínio cognitivo do estudante, que nessa taxonomia é composto pela Dimensão do Processo Cognitivo (DPC) e pela Dimensão do Conhecimento (DC). Ao optar pelo sequenciamento de ações pedagógicas moldadas segundo o perfil cognitivo do estudante, a esta proposta evita a 
necessidade de conhecer a estrutura curricular ou o encadeamento de conteúdo, pois o objetivo do sequenciamento se dá no nível do domínio cognitivo do estudante, conforme definido pela TB. Para tornar isso viável, nesta proposta o perfil do estudante é modelado sob a perspectiva de uma das dimensões que compõem ASSIST, denominada Revised Approaches to Studying Inventory $(R A S I)$.

O desenvolvimento das teorias da Taxonomia de Bloom e do RASI se baseiam no fato de que o progresso cognitivo ocorre de aspectos concretos para abstratos, que podem ser habilidades ou conhecimentos que o estudante adquire durante o processo de aprendizagem. A partir desse ponto em comum entre as duas teorias, como contribuição direta desse trabalho, foi definida uma relação entre as ações pedagógicas modeladas segundo a hierarquia da Taxonomia de Bloom e o perfil RASI.

Para desenvolver esta proposta, inicialmente foi coletado o perfil $R A S I$ do estudante por meio de um questionário. A partir disso, foi utilizado o AG para realizar o sequenciamento das ações pedagógicas estruturadas segundo a hierarquia da Taxonomia de Bloom e personalizadas de acordo com o perfil RASI do estudante. A personalização do sequenciamento foi possível, graças ao mapeamento realizado entre as teorias Taxonomia de Bloom e RASI, pois viabilizou a definição de índices $R A S I$ para as sequências de ações, comparáveis aos índices do perfil $R A S I$ de estudantes. Sendo assim, nos experimentos realizados é analisada a qualidade a personalização do sequenciamento, sob o ponto de vista da similaridade entre perfis $R A S I$ de estudantes e sequências de ações pedagógicas.

Neste trabalho, o problema do sequenciamento de ações pedagógicas é tratado sob a ótica do PA, de modo que o objetivo é determinar uma sequência de ações a partir de um estado inicial, que é o perfil RASI do estudante. Para isso, esse problema é abordado usando um Algoritmo Genético (AG) e sua função de aptidão (fitness) incorpora o resultado do mapeamento entre a Taxonomia de Bloom e o RASI, sendo outra contribuição deste trabalho. Os resultados são promissores e instigam explorar o refinamento do método proposto e realizar experimentos em ambiente real, para uma avaliação mais abrangente.

$\mathrm{Na}$ Seção 2, é realizada uma resenha literária acerca do PA sob a perspectiva do uso de computação bioinspirada, além disso, são tratados conceitos importantes para o desenvolvimento deste trabalho, em relação à Taxonomia de Bloom e ao RASI. Na Seção 3, é apresentado o mapeamento entre RASI e Taxonomia de Bloom. Na Seção 4, é exposto o modelo proposto, abordando um exemplo de utilização e a implementação do Algoritmo Genético. Na Seção 5, são apresentados os experimentos realizados, além da avaliação da proposta a partir dos resultados obtidos. Na Seção 6, são abordadas as conclusões, as vantagens e as fragilidades desta proposta, além de trabalhos futuros.

\section{Revisão da Literatura}

Para a realização do processo de sequenciamento de ações pedagógicas, é necessária a ponderação de diversos elementos, como o planejador adequado para esse sequenciamento, por exemplo. Além disso, a modelagem das ações e a definição das características do estudante podem ser fundamentais para o sucesso da abordagem. Tais questões são discutidas nas próximas subseções.

\subsection{Planejamento Automatizado Apoiado por Computação Bioinspirada}

O uso de algoritmos bioinspirados para realização do planejamento automatizado é viável no contexto de sequenciamento pedagógico. Dentre as abordagens bioinspiradas, o Algoritmo Otimização de Colônia de Formigas foi explorado em (Rastegarmoghadam \& Ziarati, 2017) e 
(Vanitha, Krishnan, \& Elakkiya, 2019). Já o Algoritmo Genético é explorado em (Lin, Chang, \& Chu, 2016), (Hssina, 2019), (de Miranda, et al., 2019) e (Vanitha, Krishnan, \& Elakkiya, 2019). Além dessas técnicas, o Algoritmo Presa-Predador é abordado em (Machado, Barrére, \& Souza, 2018). Nesses trabalhos, a abordagem bioinspirada é utilizada sob a perspectiva de problemas de otimização no contexto de sequenciamento de ações pedagógicas.

Os problemas de otimização abordados no cenário de aprendizagem são diversos, dentre os quais podemos destacar: i) A busca pela rota de aprendizagem ideal, de acordo com o perfil do estudante, observada em (Rastegarmoghadam \& Ziarati, 2017), (Hssina, 2019) e (Vanitha, Krishnan, \& Elakkiya, 2019); ii) o desempenho do estudante e o tempo de aprendizagem, abordados em (Lin, Chang, \& Chu, 2016); iii) o sequenciamento curricular, como visto em (Agbonifo \& Olanrewaju, 2018); iv) a satisfação do estudante, além da minimização e equalização no tempo de trabalho entre os participantes de um grupo são tratados em (de Miranda, et al., 2019).

Diante dos trabalhos supracitados, constata-se que o sequenciamento de ações pedagógicas pode ser interpretado como um problema de otimização. Assim, é viável a utilização de algoritmos bioinspirados para esse tipo de problema (Hssina, 2019). A grande disponibilidade de parâmetros justifica o uso dessa estratégia, pois a manipulação desses por meio de técnicas como o PA via PDDL, por exemplo, exigiriam alto esforço computacional e inviabilizariam tal aplicação (Brie \& Morignot, 2005). Baseado no fato de que a maioria dos trabalhos encontrados na literatura adotam o uso de AG para o PA, apresentando bons resultados, neste trabalho também se adotou o uso dessa técnica.

\subsection{Taxonomia de Bloom}

Conforme ponderado em (Costa, Pereira Júnior, \& Fernandes, 2019), as ações pedagógicas adotadas nos trabalhos analisados baseiam-se majoritariamente em conteúdos específicos por meio do sequenciamento de Objetos de Aprendizagem (OAs). Com isso, é relatado que estratégicas focadas no processo cognitivo do estudante podem trazer vantagens. Uma alternativa apontada nesse trabalho é a utilização da Taxonomia de Bloom, pois apresenta uma análise bidimensional da evolução da aprendizagem, considerando a Dimensão do Processo Cognitivo e a Dimensão do Conhecimento, com o objetivo de auxiliar no planejamento didático-pedagógico. Essa estrutura é apresentada na Tabela 1.

Tabela 1: Representação bidimensional da Taxonomia Revisada de Bloom.

\begin{tabular}{|c|c|c|c|c|c|c|c|}
\hline & \multicolumn{6}{|c|}{ Dimensão do Processo Cognitivo } \\
\hline & & Lembrar & Compreender & Aplicar & Analisar & Avaliar & Criar \\
\hline \multirow{4}{*}{ 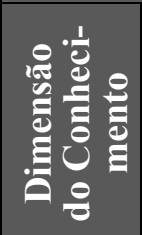 } & Factual & & & & & & \\
\hline & Conceitual & & & & & & \\
\hline & Procedural & & & & & & \\
\hline & Metacognitivo & & & & & & \\
\hline
\end{tabular}

Fonte: Adaptado de (Krathwohl, 2002).

Na Taxonomia de Bloom, a Dimensão do Processo Cognitivo é composta por seis estados cognitivos hierarquicamente estruturados a partir de Lower Order Cognitive Skills (LOCS) para Higher Order Cognitive Skills (HOCS). Cada um desses estados é associado a um conjunto de ações que podem ser desenvolvidas pelo estudante no contexto da aprendizagem. Já a Dimensão do Conhecimento, diz respeito ao conhecimento adquirido ou produzido pelo estudante e é estruturada em quatro níveis a partir do nível mais concreto, para o nível mais abstrato. Nessa estrutura, cada célula na Tabela 1 representa um objetivo educacional. 
Os estados cognitivos na Dimensão do Processo Cognitivo são compostos por um significado e verbos chave no gerúndio. Tais verbos são representativos das ações esperadas em cada estado, como se segue:

- Lembrar: Diz respeito a recuperação de informação de longo prazo na memória. Nesse sentido, os verbos são reconhecendo e recordando;

- Compreender: Estabelecimento de significado às mensagens instrucionais, envolvendo comunicação oral, escrita e gráfica. Assim, os verbos que compõem esse estado são interpretando, exemplificando, classificando, resumindo, inferindo, comparando e explicando;

- Aplicar: Pautado na prática de determinado procedimento em um contexto específico. Nesse aspecto, os verbos representativos são executando e implementando;

- Analisar: Divisão de um conceito em partes com o intuito de evidenciar as interrelações entre si e com uma estrutura mais geral. Assim, os verbos representativos são diferenciando, organizando e atribuindo;

- Avaliar: Realização de julgamentos a partir de critérios e padrões. Assim, os verbos que constituem tal estado são verificando e criticando;

- Criar: Associar elementos de modo a constituir um novo com coerência ou originalidade. Os verbos significativos são gerando, planejando e produzindo.

A partir da definição na Dimensão do Processo Cognitivo sobre o que é esperado em termos de aprendizagem do estudante, a Dimensão do Conhecimento estrutura o conhecimento a partir do processo de desenvolvimento da aprendizagem em relação ao conteúdo (Ferraz \& Belhot, 2010). A DC é composta por 4 níveis de conhecimento transversais ao assunto, são eles:

- Factual: Elementos básicos necessários à compreensão de um conteúdo. Assim, são esperados conhecimento de terminologia e de detalhes, além de elementos específicos;

- Conceitual: Inter-relacionamento entre conceitos básicos de maneira coerente. Nesse sentido, observa-se classificações, categorias, princípios, generalizações, teorias, modelos e estruturas;

- Procedural: Como fazer algo a partir de métodos de investigação e fundamento para utilização de habilidades, algoritmos, técnicas e critérios específicos;

- Metacognitivo: Identificação da cognição em geral e da consciência do reconhecimento da própria cognição. Desse modo, são esperados os conhecimentos estratégicos, sobre as tarefas cognitivas e autoconhecimento.

Considerando esse cenário, observa-se a viabilidade de utilização da TB como elemento para a definição de ações pedagógicas. Tal constatação é observada em (Krathwohl, 2002), ao trazer a definição dos objetivos educacionais sob a ótica bidimensional, onde a DC relaciona-se ao que se espera e a DPC a como se desenvolve o processo de aprendizagem do estudante. Além disso, apesar de não fazer parte do escopo, mas ser uma proposta de evolução deste trabalho, outra revisão da TB, denominada Taxonomia de Bloom Digital e proposta em (Churches, 2010), complementa a Taxonomia de Bloom definindo atividades digitais vinculadas objetivos educacionais, logo, a proposta deste trabalho vai ao encontro de tais perspectivas, pois oferece um arcabouço que pode automatizar e personalizar a recomendação dessas atividades considerando o perfil RASI do estudante.

\subsection{Definição do Perfil do Estudante Usando RASI}

O ASSIST apresentado em (Entwistle \& Tait, 2013) oferece uma análise do processo de aprendizagem do estudante considerando três dimensões: i) O que é aprender; ii) Abordagens para 
estudar; iii) Preferências para diferentes tipos de curso e ensino. A coleta de informações em cada uma dessas dimensões é realizada por meio de respostas a questionários. Esse método é interessante, pois pode fornecer objetivamente uma visão de aspectos relacionados ao processo de aprendizagem do estudante.

Tabela 2: Subescalas das categorias RASI.

\begin{tabular}{|l|l|l|}
\multicolumn{1}{|c|}{ Surface } & \multicolumn{1}{|c|}{ Strategic } & \multicolumn{1}{c|}{ Deep } \\
\hline - Falta de propósito. & - Estudo organizado. & - Busca por significado. \\
- Memorização não relacionada. & - Gerenciamento de tempo. & - Relacionamento de ideias. \\
- Medo do fracasso. & - Realização. & - Uso de evidência. \\
- Limite do plano de estudos. & $\begin{array}{l}\text { - Prontidão para demandas de } \\
\text { avaliação. }\end{array}$ & $\begin{array}{l}\text { - Interesse em ideias. } \\
\text { - Monitoramento da eficácia. }\end{array}$ \\
\hline
\end{tabular}

Fonte: Adaptado de (Richardson, 2000).

A dimensão ii) Abordagens para estudar, utiliza o Revised Approaches to Studying Inventory (RASI), que é detalhado em (Richardson, 2000) para definir o perfil cognitivo, ou perfil RASI, do estudante em relação à aprendizagem. Essa teoria utiliza um questionário com 52 questões na escala Likert ${ }^{1}$ com 5 níveis e tem a finalidade de medir as atitudes do estudante sobre a abordagem à aprendizagem. Assim, esse perfil é dado por três eixos e cada um desses é composto por subescalas que os descrevem. Para cada uma dessas subescalas foram definidas 4 questões. Tais subescalas são apresentadas na Tabela 2.

\begin{tabular}{|c|c|c|c|c|c|c|}
\hline \multirow[b]{4}{*}{ LOCS } & & & CONCRETC & $\int<-=$ & ---- & $\Rightarrow$ ABSTRATO \\
\hline & & & \multicolumn{4}{|c|}{ Dimensão do Conhecimento } \\
\hline & & & Factual & Conceitual & Procedural & Metacognitivo \\
\hline & \multirow{6}{*}{ 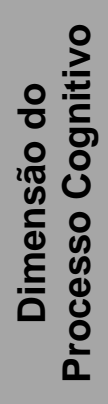 } & Lembrar & ação 1 & ação 2 & ação 3 & ação 4 \\
\hline \multirow{4}{*}{$\begin{array}{l}1 \\
1 \\
1 \\
1 \\
1\end{array}$} & & Compreender & ação 5 & ação 6 & ação 7 & ação 8 \\
\hline & & Aplicar & ação 9 & ação 10 & ação 11 & ação 12 \\
\hline & & Analisar & ação 13 & ação 14 & ação 15 & ação 16 \\
\hline & & Avaliar & ação 17 & ação 18 & ação 19 & ação 20 \\
\hline HOCS & & Criar & ação 21 & ação 22 & ação 23 & ação 24 \\
\hline
\end{tabular}

Figura 1: Estruturação de ações pedagógicas a partir da Taxonomia de Bloom.

A partir da estrutura mostrada na Tabela 2, trabalhos como os propostos em (Brown, White, Wakeling, \& Naiker, 2015) e (Shang, 2019) corroboram a perspectiva de que o perfil RASI do estudante apresenta uma hierarquia variando de LOCS para HOCS. Nesse sentido, em (Brown, White, Wakeling, \& Naiker, 2015) é apresentada uma aderência entre as subescalas da categoria Deep e os estados cognitivos da Dimensão do Processo Cognitivo na Taxonomia de Bloom. Desse modo, a busca por um método que possibilite o mapeamento entre o RASI e a Taxonomia de Bloom pode expandir as possibilidades de uso dessas teorias e trazer benefícios no contexto de

\footnotetext{
${ }^{1}$ Likert, R. (1932). A technique for the measurement of attitudes. Archives of psychology.
} 
recomendações pedagógicas baseadas em ações modeladas a partir do perfil cognitivo do estudante.

\section{Mapeamento da Relação entre RASI e Taxonomia de Bloom}

Como apresentado na Subseção 2.2, a Taxonomia de Bloom oferece um arcabouço significativo no contexto da estruturação de ações pedagógicas considerando objetivos educacionais delimitados pelo processo cognitivo e pelo conhecimento durante o desenvolvimento da aprendizagem do estudante. A partir dos elementos fornecidos pela análise bidimensional na Taxonomia de Bloom, neste trabalho foram propostas ações pedagógicas representativas da Dimensão do Processo Cognitivo e da Dimensão do Conhecimento, conforme arranjo apresentado na Figura 1.

Nesse aspecto, segundo (Krathwohl, 2002), é observada a possibilidade de flexibilização na hierarquia da Taxonomia de Bloom em detrimento da percepção do perfil do estudante. Com isso, considerando as 24 ações propostas conforme Figura 1, existem 16.777.216 de possibilidades de arranjos dessas ações. Note que pela definição da TB, a quantidade de objetivos educacionais pode ser variável, porém as restrições hierárquicas $L O C S / H O C S$ ou concreto/abstrato devem ser respeitadas. Portanto, uma sequência de ações pedagógicas pautadas por tal hierarquia também deve respeitar tais restrições. Desse modo, tal abordagem proporciona gerar sequências de ações sempre válidas, desde que respeitadas tais premissas.

Outro ponto a ser destacado é que na estrutura proposta na Figura 1, cada estado cognitivo da Dimensão do Processo Cognitivo é representado por um conjunto de ações que representam cada nível da DC. Nesse sentido, um conjunto que representa o estado cognitivo é composto 4 ações. Assim, cada ação possui uma relevância, ou peso $p=1 / 4$. O peso de cada estado cognitivo $\left(P_{\text {estado_cognitivo }}\right)$ é o somatório dos pesos $p$ de cada ação, logo $P_{\text {estado_cognitivo terá o valor }}$ máximo de 1 . Tal ponderação será importante na Seção 4 para realizar o cálculo do grau de relevância de uma sequência de ações para um perfil, de modo a incluir a análise bidimensional da TB.

Conforme discutido nas subseções 2.2 e 2.3, tanto a Taxonomia de Bloom quanto o RASI, apresentam uma evolução em relação ao estado cognitivo do estudante, variando de LOCS para HOCS. Tais teorias apresentam um ponto de convergência ao explorar a representação do processo cognitivo num espectro evolutivo partindo de elementos concretos para abstratos, conforme relatado em (Brown, White, Wakeling, \& Naiker, 2015) e (Shang, 2019). Assim, neste trabalho foi proposto o mapeamento entre o perfil $R A S I$ e a Taxonomia de Bloom, elaborado a partir da classificação de cada uma das 52 questões dadas pelo $R A S I$, de acordo com sua aderência aos estados cognitivos definidos pela Taxonomia de Bloom. Isso foi realizado, considerando os verbos chave que compõem cada um dos estados cognitivos na Taxonomia de Bloom e suas respectivas definições. Na Tabela 3 é apresentado esse mapeamento utilizando o número identificador de cada questão RASI, tal qual enumerada em (Entwistle \& Tait, 2013). 
Tabela 3: Mapeamento entre questionário RASI e a Dimensão do Processo Cognitivo da Taxonomia de Bloom.

\begin{tabular}{|c|c|c|c|c|c|c|c|}
\hline & \multicolumn{6}{|c|}{ Dimensão do Processo Cognitivo na Taxonomia de Bloom } \\
\hline & & Lembrar & Compreender & Aplicar & Analisar & Avaliar & Criar \\
\hline \multirow{3}{*}{ 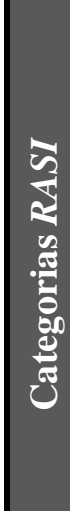 } & $\frac{8}{5}$ & $\begin{array}{c}3,8,12,16 \\
19,32,35,42 \\
45,48\end{array}$ & 6,25 & -- & 29,38 & -- & 22,51 \\
\hline & है & 27 & 37,41 & $18,31,50$ & $1,15,28,44$ & $\begin{array}{c}2,5,10,14, \\
24\end{array}$ & 40 \\
\hline & ๑ั & 26 & $4,11,52$ & -- & $\begin{array}{c}17,21,23, \\
34,36,43,49\end{array}$ & $\begin{array}{c}7,9,20,30 \\
39,47\end{array}$ & $13,33,46$ \\
\hline
\end{tabular}

No trabalho de (Shang, 2019), é explicitada a vinculação entre as subescalas RASI na categoria Deep e os estados cognitivos da Dimensão do Processo Cognitivo na Taxonomia de Bloom. Em (Brown, White, Wakeling, \& Naiker, 2015), é mencionada uma convergência entre a categoria Surface e a Taxonomia de Bloom, apesar de não ter sido realizado um mapeamento de fato. Em contraste, neste trabalho foram comparadas as questões associadas à Dimensão do Processo Cognitivo com o mapeamento proposto em (Shang, 2019). Apesar das metodologias adotadas serem distintas, a similaridade do mapeamento entre essas propostas para a categoria Deep foi de 58,3\%. Tal comparação evidencia certo grau de correlação entre a estratégia adotada neste trabalho e a proposta apresentada em (Shang, 2019). Diante disso, o refinamento desse mapeamento é uma questão a ser explorada em trabalhos futuros, considerando inclusive os resultados da corrente proposta. A partir do mapeamento expresso na Tabela 3, foi calculado um índice de relevância para cada um dos eixos da Dimensão do Processo Cognitivo em relação às categorias $R A S I$, conforme apresentado na Figura 2.

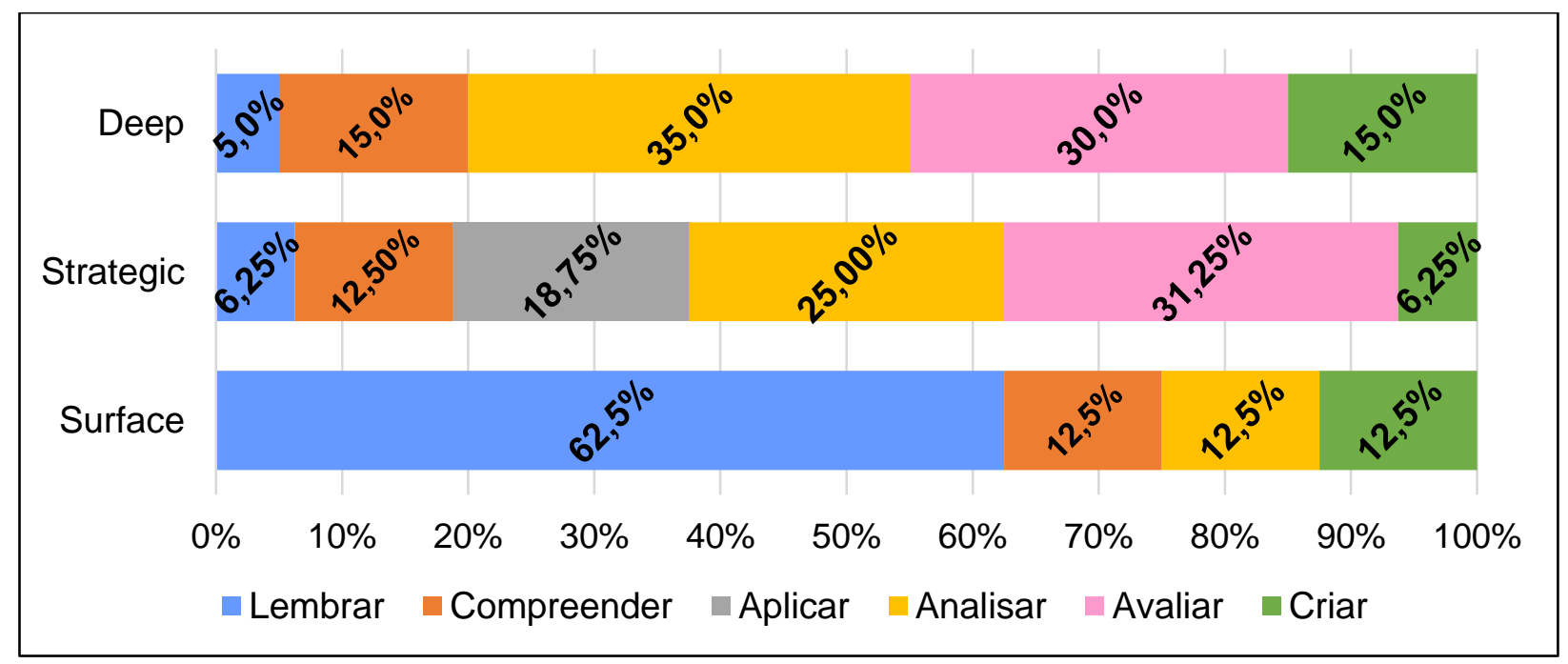

Figura 2: Taxa de relevância entre os eixos da Dimensão do Processo Cognitivo e as categorias RASI.

O mapeamento apresentado na Figura 2 permite estabelecer uma relação numérica entre os estados cognitivos da Dimensão do Processo Cognitivo e as categorias RASI. Ainda nesse sentido, é possível observar quais estados cognitivos são mais ou menos determinantes na composição dessas categorias, podendo categorizá-los com grau de relevância baixo, moderado ou alto, por 
exemplo. Essa contribuição pode apresentar benefícios no cenário de recomendações pedagógicas, uma vez que permite mensurar a relevância de uma sequência de ações estruturadas pela Taxonomia de Bloom em relação a um perfil de estudante, modelado pelo RASI. Com isso, firma-se a possibilidade de realizar tal classificação, com vistas ao sequenciamento pedagógico automatizado e personalizado de acordo com o perfil do estudante. Note que as taxas expressas na Figura 2 foram normalizadas para melhor compreensão do gráfico, entretanto, na aplicação prática desse mapeamento, é desejável a utilização dos índices $D$. A ordenação de tais índices segue a hierarquia proposta pela TB, ou seja, Lembrar, Compreender, Aplicar, Analisar, Avaliar e Criar. Desse modo, esses índices por categoria RASI são:

- $\quad D_{\text {surface }}=\langle 0.6250 ; 0.1250 ; 0.0000 ; 0.1250 ; 0.0000 ; 0.1250\rangle$

- $D_{\text {strategic }}=\langle 0.0625 ; 0.1250 ; 0.1875 ; 0.2500 ; 0.3125 ; 0.06250\rangle$

- $D_{\text {deep }}=\langle 0.0500 ; 0.1500 ; 0.0000 ; 0.3500 ; 0.3000 ; 0.1500\rangle$

$\mathrm{Na}$ Taxonomia de Bloom, a recomendação pedagógica ocorre a partir da seleção de objetivos educacionais, geralmente realizada por docente de forma manual e não adaptada ao perfil do estudante. Tal situação é imposta, dada a complexidade de individualização de recomendações pedagógicas sob essa ótica, considerando um conjunto de estudantes, como em uma sala de aula, por exemplo. Na maior parte dos casos, as diretrizes desse tipo de recomendação são pautadas pelos requisitos de curso, havendo necessidade de conhecer o encadeamento entre conteúdos. Neste trabalho, a partir deste mapeamento proposto, é possível identificar uma função para avaliar sequências de ações pedagógicas que levem em consideração a Taxonomia de Bloom e o RASI.

\section{Sequenciamento de Ações usando Algoritmo Genético}

Nesta seção, é apresentada a abordagem de sequenciamento de ações pedagógicas por meio de AG, a partir do contexto anteriormente discutido. Na Subseção 2.2 foi discutida a Taxonomia de Bloom como um método viável para estruturar ações pedagógicas que podem se relacionar com o processo cognitivo do estudante. A partir disso, na Subseção 2.3, o perfil do estudante é discutido sob a perspectiva do $R A S I$ e sua aderência à Dimensão do Processo Cognitivo. Nesse sentido, na Seção 3 foi proposto o mapeamento entre o perfil RASI e os estados cognitivos descritos na Taxonomia de Bloom. Tal arcabouço se apresenta como uma alternativa para o sequenciamento de ações pedagógicas adequadas ao perfil do estudante. Como exemplo, considere os seguintes elementos:

- Sequência de ações de acordo com a Taxonomia de Bloom:

Sequência 1: $\langle 1-2-4-5-6-7-8-15-17-18-19-20-22-24\rangle$

- Perfil RASI do estudante:

Perfil $A$ : $\langle\mathrm{su}=0.34375 ;$ st $=0.5 ;$ de $=0.65\rangle$

onde: $s u=$ surface; st $=$ strategic; de $=$ deep.

O cálculo dos índices $R A S I(R=\langle s u$; st; de $\rangle)$ para a Sequência 1 é dado pelo somatório do produto entre o peso de cada estado cognitivo ( $P_{\text {estado_cognitivo }}$ ) e o índice da categoria $R A S I$ $\left(D_{\text {categoria }}\right)$, definidos na Seção 3 . Note que o peso $\left(P_{\text {estado_cognitivo }}\right)$ somente terá valor máximo (1), quando as 4 ações definidas para aquele estado cognitivo forem sequenciadas, caso contrário, o valor será múltiplo de 0.25 . Por exemplo, para a Sequência 1 , os pesos de cada estado cognitivo são, respectivamente, $P=\langle 0.75 ; 1.0 ; 0 ; 0.25 ; 1.0 ; 0.5\rangle$. Logo, os índices $R A S I R$ para Sequência 1 são: $R=\langle\mathrm{su}=0.6875$; st $=0.578125$; de $=0.65\rangle$. Note que a partir desse resultado é possível estabelecer uma comparação entre os índices do Perfil $A$ e os índices $R A S I$ em $R$. 
A partir do contexto supracitado, o planejamento automatizado, apoiado por AG pode ser uma alternativa viável para realizar o sequenciamento de ações pedagógicas personalizadas. Assim, neste trabalho, tais recursos são utilizados para sequenciar ações pedagógicas modeladas pela Taxonomia de Bloom e personalizadas de acordo com o perfil RASI do estudante.

\subsection{Sequenciamento de Ações Pedagógicas Usando AG}

Neste trabalho, a modelagem das ações pedagógicas é realizada segundo a Taxonomia de Bloom, discutida na Seção 3. Além disso, tal modelagem faz uso das condições oferecidas pelo PA, pois o sequenciamento de ações pedagógicas leva em consideração um objetivo pedagógico a ser alcançado. Desse modo, o estado inicial do planejamento é a identificação do perfil do estudante e o objetivo desse planejamento é encontrar uma sequência de ações aderente a esse perfil. A implementação do AG, considerou os parâmetros estabelecidos em (Engelbrecht, 2007). Assim, foi criada uma população inicial, em seguida os indivíduos dessa população foram avaliados até que o critério de parada fosse atingido. Durante esse processo, os operadores genéticos de mutação e de cruzamento também foram adotados, conforme detalhes discutidos nesta subseção.

No AG proposto, o indivíduo é representado por uma sequência de 24 bits, lidos da esquerda para a direita. Esses representam cada uma das possíveis ações da Taxonomia de Bloom,

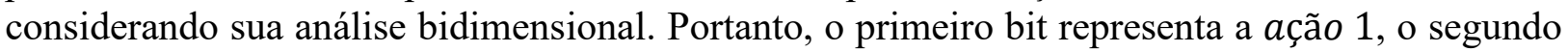
bit a $a$ ção 2 e assim por diante, até o $24^{\circ}$ bit, que representa a ação 24 , conforme as ações estruturadas na Figura 1. Quando o valor de um bit for 1, significa que aquela ação fará parte do sequenciamento e quando esse valor for zero 0 , tal ação não estará presente no sequenciamento. Observa-se também que o indivíduo tem tamanho fixo de 24 bits, porém a sequência de ações pedagógicas tem tamanho variável, pois apenas os bits com valor 1 representam as ações que serão sequenciadas. Um exemplo do indivíduo é mostrado na Figura 3.

\begin{tabular}{|c|c|c|c|c|c|c|c|c|c|c|c|c|}
\hline TB & \multicolumn{4}{c|}{ Lembrar } & \multicolumn{4}{c|}{ Compreender } & \multicolumn{4}{c|}{ Aplicar } \\
\hline Posição & $\mathbf{1}^{\circ}$ & $2^{\circ}$ & $\mathbf{3}^{\circ}$ & $\mathbf{4}^{\circ}$ & $\mathbf{5}^{\circ}$ & $\mathbf{6}^{\circ}$ & $\mathbf{7}^{\circ}$ & $\mathbf{8}^{\circ}$ & $\mathbf{9}^{\circ}$ & $\mathbf{1 0}^{\circ}$ & $\mathbf{1 1}^{\circ}$ & $\mathbf{1 2}^{\circ}$ \\
\hline Valor & 1 & 1 & 0 & 1 & 1 & 0 & 0 & 1 & 0 & 0 & 1 & 1 \\
\hline
\end{tabular}

\begin{tabular}{|c|c|c|c|c|c|c|c|c|c|c|c|c|}
\hline TB & \multicolumn{4}{c}{ Analisar } & \multicolumn{4}{c|}{ Avaliar } & \multicolumn{4}{c|}{ Criar } \\
\hline Posição & $13^{\circ}$ & $14^{\circ}$ & $15^{\circ}$ & $16^{\circ}$ & $17^{\circ}$ & $1^{\circ}$ & $19^{\circ}$ & $2^{\circ}$ & $2^{\circ}$ & $2^{\circ}$ & $2^{\circ}$ & $2^{\circ}$ \\
\hline Valor & 1 & 0 & 0 & 1 & 0 & 0 & 1 & 1 & 0 & 0 & 1 & 1 \\
\hline
\end{tabular}

Figura 3: Representação binária do indivíduo no AG.

A modelagem do indivíduo, conforme apresentada na Figura 3, sempre gera uma sequência de ações válidas. Note que na Taxonomia de Bloom, a hierarquia dos estados cognitivos pode ser adequada às necessidades do estudante (Krathwohl, 2002). Nessa hierarquia é desejável que ações menos complexas sejam recomendadas antes de ações mais complexas. Em outras palavras, esta premissa implica na necessidade de recomendar ações, representadas no indivíduo por cada posição cujo bit tenha valor verdadeiro, seguindo a ordem da esquerda para a direita, desse modo respeitando tal restrição imposta pela hierarquia da Taxonomia de Bloom.

$\mathrm{Na}$ primeira etapa do $\mathrm{AG}$ proposto, é criada a população com a quantidade $\max _{i}$ de indivíduos que representam potenciais soluções. Esses são gerados aleatoriamente e codificados em uma matriz binária, onde cada linha possui 24 bits. Cada bit de um indivíduo representa uma ação de acordo com a estrutura apresentada na Figura 3. 
A função de aptidão (fitness) é dada pela Eq. 1 e utiliza os índices RASI surface (su), strategic (st) e deep (de) relativos à sequência de ações gerada pelo AG e ao perfil do estudante. Para a sequência de ações, esses índices são calculados conforme descrito na Seção 3. Para o perfil do estudante, esses índices são obtidos por meio do questionário $R A S I$, conforme descrito na Subseção 2.3.

$$
\text { fitness }(I)=\operatorname{distancia}(I, E)+\text { penalidade }(I, E)
$$

onde $I$ e $E$ representam os índices $R A S I$ su, st e de da sequência de ações e do perfil do estudante, respectivamente. Esse problema de otimização envolve três variáveis correspondentes aos índices su, st e de, que são maiores ou iguais a zero. O problema então, consiste em encontrar uma sequência de ações de tal modo que o fitness seja o menor possível. A função fitness é composta pelo cálculo da Distância Euclidiana calculada entre os índices $R A S I$ da sequência de ações e do perfil do estudante, conforme Eq. 2, além de uma penalidade estabelecida a partir de uma ordem de relevância entre os índices RASI, conforme descrita a seguir.

$$
\operatorname{distancia}(I, E)=\sqrt{(\operatorname{su}(I)-s u(E))^{2}+(s t(I)-s t(E))^{2}+(\operatorname{de}(I)-\operatorname{de}(E))^{2}}
$$

A penalidade $(I, E)$ é calculada considerando a comparação da ordem de relevância dos índices $R A S I$ entre $I$ e $E$. A cada índice é atribuído um peso 3, 2 ou 1, conforme sua relevância, sendo 3 para o mais relevante e 1 para o menos. Desse modo a penalidade é a soma do resultado de (distancia $(I, E) / 6 *$ peso) para cada um dos índices com relevância discrepante.

Utilizando o Perfil A e a Sequência 1, dados como exemplos nesta seção, temos a ordem de relevância mostrada na Tabela 4.

Tabela 4: Exemplo da ordem de relevância dos índices $R A S I$.

\begin{tabular}{|l|c|c|c|c|c|c|}
\hline & \multicolumn{3}{|c|}{ Índices RASI } & \multicolumn{3}{c|}{ Pesos / Ordem de relevância } \\
\cline { 2 - 7 } & su & st & de & Peso 3 & Peso 2 & Peso 1 \\
\hline Indivíduo $(I)$ & 0.687500 & 0.578125 & 0.65000 & $s u$ & de & st \\
\hline Estudante $(E)$ & 0.343750 & 0.500000 & 0.65000 & de & st & > \\
\hline
\end{tabular}

Nesse exemplo, a penalidade é calculada considerando os pesos 3, 2 e 1, pois a ordem de relevância para todos os índices é divergente. Logo temos:

$$
\begin{aligned}
& \text { penalidade }(I, E)= \\
& \quad \operatorname{distancia}(I, E) / 6 * 3+\operatorname{distancia}(I, E) / 6 * 2+\operatorname{distancia}(I, E) / 6
\end{aligned}
$$

A partir disso, exemplos de cálculos para distancia, penalidade e fitness são:

- $\operatorname{distancia}(I, E)=$

$$
\sqrt{(0.6875-0.34375)^{2}+(0.578125-0.5)^{2}+(0.65-0.65)^{2}}=0.352516
$$

- $\quad$ penalidade $(I, E)=0.352516 / 6 * 3+0.352516 / 6 * 2+0.352516 / 6=0.352516$

- $\operatorname{fitness}(I)=\operatorname{distancia}(I, E)+$ penalidade $(I, E)=0.705032$

Note que a penalidade pode, no máximo, dobrar o valor dado pela distância euclidiana, como nesse exemplo, em que todos os índices RASI apresentam ordem de relevância divergente.

O critério de parada do AG ocorre quando o número máximo de gerações (max_g) é atingido ou o fitness do indivíduo tem o valor zero. A seleção dos indivíduos que comporão a próxima geração da população é obtida por meio de torneio. Para isso, é feita a seleção aleatória de três 
indivíduos da população, optando pela escolha do indivíduo mais apto, segundo uma probabilidade $k_{-} t$. Essa abordagem pode reduzir o peso dos indivíduos mais adaptados para o algoritmo e proporcionar maior diversidade à população.

O cruzamento no AG é realizado considerando blocos de 4 bits, dadas as 6 dimensões da Taxonomia de Bloom que estes blocos representam. A quantidade de blocos e quais os blocos serão permutados são determinados aleatoriamente. Após essa etapa, caso o fitness do novo indivíduo seja melhor do que pelo menos um dos indivíduos que o geraram, este torna-se apto a compor o ranqueamento para formação da nova população. Caso contrário, o novo indivíduo é descartado. No ranqueamento realizado entre a população atual e os novos indivíduos gerados pelo cruzamento, são selecionados os max_ $i$ indivíduos mais aptos para fazerem parte da nova população. Após a geração da nova população, é realizada a mutação com taxa $k \_m$ de indivíduos da população. A mutação realiza a inversão aleatória de 1 bit de cada um dos blocos de bits que compõem o indivíduo.

\section{Experimentos e Resultados}

Os experimentos foram realizados com o objetivo de verificar se o sequenciamento automático de ações pedagógicas apresenta índices RASI adequados ao perfil do estudante. Para tal, os experimentos foram divididos em duas etapas, a saber:

- Etapa 1: Aplicação do questionário RASI a estudantes, com o intuito de formar uma base de perfis que serão utilizados para o sequenciamento de ações pedagógicas.

- Etapa 2: Realização do sequenciamento de ações pedagógicas utilizando o método proposto, com o intuito de observar o quão adequadas são tais sequências, considerando os parâmetros propostos.

O público-alvo participante da Etapa 1 foi composto por estudantes do ensino superior na área de Tecnologia da Informação, matriculados nas instituições Universidade Federal de Uberlândia e Instituto Federal Goiano. Ao todo, 41 estudantes participaram dessa etapa.

$\mathrm{Na}$ Etapa 2, foram definidos, a partir de testes exploratórios, o tamanho da população $\max _{-} i=1000$ e o número de gerações $\max _{-} g=100$ para o AG proposto. $\mathrm{O}$ cruzamento ocorreu utilizando a quantidade aleatória de até 5 pontos, conforme descrito na Subseção 4.1 e a probabilidade $k_{-} t$ de seleção por torneio dos indivíduos foi de $60 \%$. A probabilidade de mutação $k \_m$, foi de $10 \%$. Outras combinações de valores para esses parâmetros não produziram alterações significativas em alguns casos e em outros, pioraram os resultados. O AG foi executado no máximo $\max _{-} g$ vezes para cada perfil. Caso seja encontrada a melhor solução antes da quantidade de execuções atingir $\max \_g$, a execução do AG é finalizada.

As taxas de similaridade entre cada perfil e a respectiva sequência de ações considerando o fitness são apresentadas na Figura 2. Observa-se que 16 sequências de ações tiveram a taxa de similaridade maior ou igual a $99 \%$, sendo que desses, uma sequência teve a taxa de $100 \%$. A maior parte das sequências, totalizando 22 , apresentou taxa de similaridade entre $92 \%$ e $98 \%$. No outro extremo dos resultados, nota-se que 3 sequências apresentaram essa taxa menor que $90 \%$, sendo que a pior foi de $88 \%$ para 1 sequência. Esses resultados apresentaram-se promissores sob o ponto de vista da função de aptidão. 


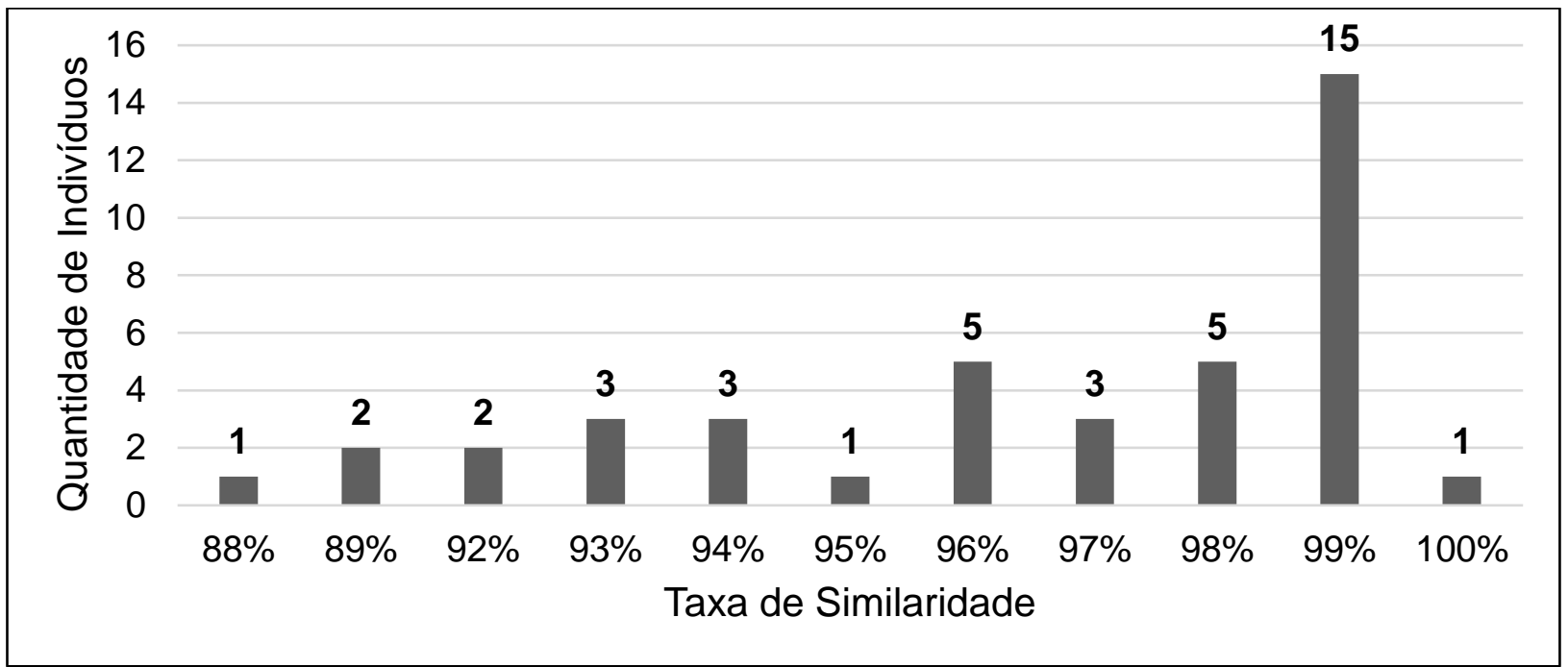

Figura 4: Taxas de similaridade entre as sequências de ações e os perfis cognitivos dos estudantes.

Outra análise realizada para auxiliar na verificação da aderência entre as sequências de ações pedagógicas e os respectivos perfis cognitivos foi conduzida a partir da observação do grau de relevância dos estados cognitivos da Taxonomia de Bloom, considerando três níveis (baixo, moderado e alto), conforme discutido na Seção 3. A partir dessa análise, temos a organização apresentada na Tabela 1.

Tabela 5: Graus de relevância entre as categorias RASI e os estados cognitivos da Taxonomia de Bloom.

\begin{tabular}{|c|c|c|c|}
\hline \multirow{2}{*}{$\begin{array}{c}\text { Categorias } \\
\text { RASI }\end{array}$} & Baixo & Moderado & Alto \\
\cline { 2 - 4 } Surface & Aplicar, Avaliar & $\begin{array}{c}\text { Compreender, } \\
\text { Analisar, Criar }\end{array}$ & Lembrar \\
\hline Strategic & Lembrar, Criar & $\begin{array}{c}\text { Compreender, } \\
\text { Aplicar }\end{array}$ & Analisar, Avaliar \\
\hline Deep & Lembrar, Aplicar & $\begin{array}{c}\text { Compreender, } \\
\text { Criar }\end{array}$ & Analisar, Avaliar \\
\hline
\end{tabular}

O mapeamento apresentado na Figura 2 distingue a taxa de relevância de cada categoria da Dimensão do Processo Cognitivo em relação a cada eixo do RASI. A estratificação dessa relação em graus de relevância produziu o resultado apresentado na Tabela 5, em que para cada eixo do $R A S I$ foram ranqueadas as categorias da DPC de acordo com o valor da taxa de relevância. Essas categorias foram classificadas duas a duas com grau de relevância baixo, moderado ou alto, seguindo a ordem de ranqueamento. Esse padrão não foi possível ser seguido para o eixo Surface, pois existem três categorias da DPC com taxas de relevância iguais. A avaliação das sequências de ações pedagógicas considerando essa classificação é observada na Tabela 6 .

Na Tabela 6, que mostra a taxa de seleção de ações de acordo com os graus de relevância presentes na Tabela 5, a taxa média geral de recomendação de atividades é maior e o desvio padrão é menor para o grau de relevância Alto. Tal resultado é satisfatório, pois corrobora a eficácia da função de aptidão do AG, uma vez que na média geral, o sequenciamento de atividades prioriza 
Tabela 6: Média e desvio padrão das taxas de seleção de ações de acordo com os graus de relevância.

\begin{tabular}{|c|c|r|r|r|}
\hline \multirow{2}{*}{ Categorias RASI / Medida } & \multicolumn{3}{|c|}{ Grau de Relevância } \\
\cline { 2 - 5 } & Média & \multicolumn{1}{|c|}{ Baixo } & \multicolumn{1}{c|}{ Moderado } & \multicolumn{1}{c|}{ Alto } \\
\hline \multirow{3}{*}{ Geral } & Desvio Padrão & $69.27 \%$ & $61.46 \%$ & $85.42 \%$ \\
\cline { 2 - 5 } & Média & 0.276 & 0.250 & 0.150 \\
\hline \multirow{3}{*}{ Surface } & Desvio Padrão & $75.69 \%$ & $50.00 \%$ & $90.28 \%$ \\
\cline { 2 - 5 } & Média & 0.289 & 0.167 & 0.125 \\
\hline \multirow{3}{*}{ Strategic } & Desvio Padrão & $50.74 \%$ & $89.71 \%$ & $62.50 \%$ \\
\cline { 2 - 5 } & Média & 0.093 & 0.173 & 0.133 \\
\hline \multirow{2}{*}{ Deep } & Desvio Padrão & $33.33 \%$ & $66.67 \%$ & $68.75 \%$ \\
\cline { 2 - 5 } & & 0.171 & 0.219 & 0.068 \\
\hline
\end{tabular}

aquelas com grau de relevância maior. Ao observar esses índices estratificados por categoria RASI, a Strategic apresenta maior taxa de recomendação para o grau Moderado. Tal situação é previsível, uma vez que considerando a definição da categoria Strategic, esta se figura como uma transição da categoria Surface para a Deep, como abordado na Seção 3, podendo apresentar situações de sobreposição às demais categorias. Compreendemos estes resultados como promissores, porém em trabalhos futuros a partir de uma base maior de casos, estima-se que farse-á necessário o refinamento do mapeamento estabelecido na Figura 2, a fim de aprimorar tais resultados.

\section{Considerações Finais}

O sequenciamento de ações pedagógicas é uma questão emergente no contexto educacional. Notase a importância de explorar técnicas que tenham como foco o processo cognitivo do estudante, para além de restrições curriculares. Assim, a modelagem de ações sob a perspectiva da Taxonomia de Bloom é interessante, pois essas são sistematizadas sob a ótica cognitiva do estudante. Além disso, a utilização do RASI para a modelagem do perfil do estudante mostra-se pertinente, pois torna viável o estabelecimento de uma relação entre tal perfil e as ações pedagógicas delineadas pela Taxonomia de Bloom.

Nota-se a praticabilidade da relação supracitada, pois em ambas as bases teóricas, o processo cognitivo do estudante é hierarquizado numa variação de $L O C S$ para $H O C S$. Desse modo, uma das contribuições deste trabalho é a expansão e o detalhamento dessa relação a partir dos trabalhos de (Brown, White, Wakeling, \& Naiker, 2015)e (Shang, 2019), por meio do mapeamento entre a abordagem bidimensional da Taxonomia de Bloom e o RASI.

O PA tem como premissa o sequenciamento de ações, de maneira eficaz, para atingir um objetivo. No contexto de recomendações pedagógicas, sua implementação a partir da linguagem $P D D L$ é interessante, pois permite a modelagem de ações bem definidas. Entretanto, constata-se que tal abordagem apresenta limitações à medida que as características do estudante são refinadas, tornando impraticáveis computacionalmente as heurísticas necessárias à busca no espaço de soluções. Uma alternativa adequada a esse problema é o PA apoiado por AG. 
Outra contribuição deste trabalho é a realização do sequenciamento de ações pedagógicas modeladas a partir do processo cognitivo do estudante por meio de planejamento automatizado apoiado por AG. Neste trabalho é possível notar a viabilidade na realização de sequenciamentos personalizados a partir da similaridade entre o perfil do estudante dado pelo RASI e sequências de ações pedagógicas modeladas pela Taxonomia de Bloom. Uma limitação deste trabalho, a ser explorada em trabalhos futuros, é incluir no modelo do estudante outras características como metadados de AVA, nível de conhecimento ou de satisfação do estudante, por exemplo. Como consequência, outras funções de aptidão poderão ser desenvolvidas.

A partir das implicações dos resultados deste trabalho, como proposta futura, objetiva-se a comparação do AG com outras técnicas de computação bioinspirada. Outra proposta futura é a implementação da seleção de atividades de acordo com a Taxonomia de Bloom Digital, a fim de recomendá-las a estudantes, para tal, far-se-á necessário o mapeamento dessas atividades em consonância com a análise bidimensional da Taxonomia de Bloom.

\section{Agradecimentos}

Os autores deste trabalho agradecem à Universidade Federal de Uberlândia (UFU) e ao Instituto Federal de Educação, Ciência e Tecnologia Goiano (IF Goiano) pelo apoio ao desenvolvimento desta pesquisa.

\section{Artigo Premiado Estendido}

Esta publicação é uma versão estendida do $3^{\circ}$ melhor artigo do Simpósio Brasileiro de Informática na Educação (SBIE) 2020, intitulado "Sequenciamento de Ações Pedagógicas por Algoritmo Genético Utilizando Taxonomia de Bloom e ASSIST”, DOI: 10.5753/cbie.sbie.2020.1273.

\section{Referências}

Agbonifo, O. C., \& Olanrewaju, A. O. (2018). Genetic Algorithm-based Curriculum Sequencing Model For Personalized E-Learning System. International Journal of Education and Management Engineering, 5, pp. 27-35. doi:10.5815/ijmecs.2018.05.04 [GS Search]

Ariyaratne, M. K., \& Fernando, T. G. (2014). A comparative study on nature inspired algorithms with firefly algorithm. International Journal of Engineering and Technology, 4, pp. 611-617. [GS Search]

Brie, A. H., \& Morignot, P. (2005). Genetic Planning Using Variable Length Chromosomes. ICAPS, (pp. 320-329). [GS Search]

Brown, S., White, S., Wakeling, L., \& Naiker, M. (2015). Approaches and study skills inventory for students (ASSIST) in an introductory course in chemistry. Journal of University Teaching \& Learning Practice, pp. 1-12. [GS Search]

Churches, A. (2010). Bloom's Digital Taxonomy. Australian School Library Association NSW Incorporated. [GS Search]

Costa, N., Pereira Júnior, C., \& Fernandes, M. (2019). Recomendação de Ações Pedagógicas Utilizando Planejamento Automático e Taxonomia Digital de Bloom. Anais do XXX Simpósio Brasileiro de Informática na Educação (SBIE 2019) (pp. 1531-1540). Brasília, Brasil: Sociedade Brasileira de Computação - SBC. doi:10.5753/cbie.sbie.2019.1531 [GS Search] 
Costa, N., Pereira Junior, C., Araújo, R., \& Fernandes, M. (2019). Application of AI Planning in the Context of e-Learning. 2019 IEEE 19th International Conference on Advanced Learning Technologies (ICALT) (pp. 57-59). Maceio, Brazil: IEEE. doi:10.1109/ICALT.2019.00021 [GS Search]

de Lima, E. S., Feijó, B., \& Furtado, A. L. (2019). Procedural Generation of Quests for Games Using Genetic Algorithms and Automated Planning. SBC-Proceedings of SBGames 2019 (pp. 495-504). Rio de Janeiro, Brasil: SBC. [GS Search]

de Miranda, P. B., Ferreira, R., Castro, M. S., Neto, G. F., Souza, S. J., Santos, L. A., \& Silva, L. L. (2019). Uma Abordagem Multiobjetivo para Recomendação de Caminhos de Aprendizagem para Grupo de Usuários. Revista Brasileira de Informática na Educação, 27(3), pp. 336-350. doi:10.5753/RBIE.2019.27.03.226 [GS Search]

Engelbrecht, A. P. (2007). Computational intelligence: an introduction (2 $2^{\mathrm{a}}$ ed.). Pretoria, South Africa: John Wiley \& Sons. [GS Search]

Entwistle, N., \& Tait, H. (2013, March). Approaches and Study Skills Inventory for Students (ASSIST) (incorporating the Revised Approaches to Studying Inventory - RASI. Edinburgh: Centre for Research on Learning and Instruction, University of Edinburgh. [GS Search]

Ferraz, A. P., \& Belhot, R. V. (2010). Taxonomia de Bloom: revisão teórica e apresentação das adequações do instrumento para definição de objetivos instrucionais. Gestão \& Produção, 17(2), pp. 421-431. doi:10.1590/S0104-530X2010000200015 [GS Search]

Garrido, A., Morales, L., \& Serina, I. (2016). On the use of case-based planning for e-learning personalization. Expert Systems with Applications, 60, pp. 1-15. doi:10.1016/j.eswa.2016.04.030 [GS Search]

Hssina, B. a. (2019). A Personalized Pedagogical Objectives Based on a Genetic Algorithm in an Adaptive Learning System. Procedia Computer Science, pp. 1152-1157. doi:10.1016/j.procs.2019.04.164 [GS Search]

Júnior, C. P., Araújo, R. D., \& Dorça, F. A. (2020). Recomendação Personalizada de Conteúdo Instrucional Complementar usando Repositório de Objetos de Aprendizagem e Recursos da Web. Anais do XXXI Simpósio Brasileiro de Informática na Educação (pp. 1293-1302). Natal, Brasil: SBC. doi:10.5753/cbie.sbie.2020.1293 [GS Search]

Krathwohl, D. R. (2002). A revision of Bloom's taxonomy: An overview. Theory Into Practice, 41(4), pp. 212-218. doi:10.1207/s15430421tip4104_2 [GS Search]

Limongelli, C., \& Sciarrone, F. (2014). Fuzzy Student Modeling for Personalization of e-Learning Courses. Learning and Collaboration Technologies. Designing and Developing Novel Learning Experiences (pp. 292-301). Springer International Publishing. doi:10.1007/978-3319-07482-5_28 [GS Search]

Lin, Y.-S., Chang, Y.-C., \& Chu, C.-P. (2016). An innovative approach to scheme learning map considering tradeoff multiple objectives. Journal of Educational Technology \& Society, 19(1), pp. 142-157. [GS Search]

Machado, M., Barrére, E., \& Souza, J. (2018). Uma Abordagem Evolutiva para o Problema de Sequenciamento Curricular Adaptativo. Simpósio Brasileiro de Informática na EducaçãoSBIE (pp. 1243-1252). Fortaleza, Brasil: SBC. doi:10.5753/cbie.sbie.2018.1243 [GS Search]

Pireva, K., \& Kefalas, P. (2018). A Recommender System Based on Hierarchical Clustering for Cloud e-Learning. Intelligent Distributed Computing XI, 53, pp. 235-245. doi:10.1007/9783-319-66379-1_21 [GS Search] 
Rastegarmoghadam, M., \& Ziarati, K. (2017). Improved modeling of intelligent tutoring systems using ant colony optimization. Education and Information Technologies, 22(3), pp. $1067-$ 1087. doi:10.1007/s10639-016-9472-2 [GS Search]

Richardson, J. T. (2000). Researching student learning: Approaches to studying in campus-based and distance education. Michigan, United States of America: Society for Research into Higher Education \& Open University Press Buckingham. [GS Search]

Shang, H. (2019). Cultural Interpretation of Deep Approach to Learning: an Empirical Analysis in a Chinese University. Proceedings of Cross-Cultural Business Conference 2019 (pp. 207218). Austria: Shaker Verlag GmbH. [GS Search]

Silva, R. C., Direne, A. I., Marczal, D., Borille, A. C., \& Guimarães, P. R. (2018). Adaptabilidade de Objetos de Aprendizagem usando Calibragem e Sequenciamento Adaptativo de Exercícios. Revista Brasileira de Informática na Educação, 26(1), pp. 70-90. doi:10.5753/rbie.2018.26.01.70 [GS Search]

Vanitha, V., Krishnan, P., \& Elakkiya, R. (2019). Collaborative optimization algorithm for learning path construction in E-learning. Computers \& Electrical Engineering, 77, pp. 325338. doi:10.1016/j.compeleceng.2019.06.016 [GS Search] 